\title{
Assessing recovery from lingual nerve injury
}

\author{
Simplifying the assessment of recovery from surgical injury to the lingual nerve \\ T. Renton, A. Thexton, S-J. Crean and M. Hankins Br Dent J 2006; 200: 569-573
}

\section{Objective}

To determine the sensitivity of conventional sensory assessment in monitoring lingual nerve recovery subsequent to third molar surgery and to evaluate if the assessment methods can be predictive of injury outcome.

\section{Method}

A prospective case series of 94 patients presenting with lingual nerve injuries evaluated using objective mechanosensory and subjective methods during the recovery period of up to 12 months.

Results

The conventional tests were often unable to diagnose the presence of injury due to variability and they were not predictive of outcome. As a result of this study, we are able to identify patients more likely to have permanent rather than temporary lingual nerve injury at four to eight weeks post injury, using patient reported subjective function. The subjective function test also minimises the requirements for specialist training or equipment providing an ideal method for general dental practice.

\section{Conclusions}

The development of these simple subjective tests may enable us to identify which patients are at risk of permanent lingual nerve injuries in the early post injury phase, thus allowing expeditious therapy when indicated.

\section{IN BRIEF}

- This study proposes a simplified method of assessing lingual nerve injuries subsequent to third molar surgery.

- Two point discrimination sensitivity and neuropathic area were the only conventional tests to reliably identify nerve injury.

- The subjective function score may identify patients at risk of permanent injury in the early post-operative period allowing expedition of their referral for specialist care.

\section{COMMENT}

The authors of this article have introduced a simple subjective sensory test (which can easily be undertaken in general practice) for use in the assessment of patients who have sustained lingual nerve injuries. The paper reports a prospective study involving 94 patients with lingual nerve injuries. It evaluates and compares the efficacy of traditional detailed sensory testing with a simple subjective assessment in predicting sensory recovery after lingual nerve injury. The authors conclude that the use of a simple subjective test will correctly predict those patients who have a profound sensory disturbance that is likely to be permanent, and those who have a less significant sensory deficit that is likely to only be temporary. The paper therefore suggests that detailed sensory testing is not required to differentiate between permanent or temporary lingual nerve injuries. The patient's subjective assessment of a sensory deficit is always paramount and must be taken into consideration when managing any patient with a lingual nerve injury. Furthermore, the use of this subjective sensory test is in keeping with current clinical guidance; in the absence of any sign of recovery by three months after injury patients should be referred to a specialist centre for testing and, if necessary, nerve repair. ${ }^{1}$ Detailed sensory testing, however, is important in establishing the severity and extent of the sensory deficit, and therefore which patients are likely to benefit from surgical intervention.

A. Loescher, Department of Oral and Maxillofacial Surgery, School of Clinical Dentistry, University of Sheffield

1. Robinson PP, Loescher A R, Smith K G. A prospective, quantitative study on the clinical outcome of lingual nerve repair. Br J Oral Max Surg 38: 255-263.

doi: 10.1038/sj.bdj.4813585 